The Astrophysical Journal, 195 :L85-L88, 1975 January 15

(c) 1975. The American Astronomical Society. All rights reserved. Printed in U.S.A.

\title{
RADIATIVE TRAPPING AND HYPERFINE STRUCTURE: HCN*
}

\author{
JOHN KWAN \\ Institute For Advanced Study \\ AND \\ Nick SCOville \\ Owens Valley Radio Observatory, and California Institute of Technology \\ Received 1974 September 12; revised 1974 October 29
}

\section{ABSTRACT}

The anomalous weakness of the $F=1 \rightarrow 1$ hyperfine component in the $J=1 \rightarrow 0$ emission of interstellar $\mathrm{HCN}$ can be caused by radiative trapping in the $J=2 \rightarrow 1$ lines. The anomaly is readily produced if the $J=1$ levels are populated largely by collisional excitation from $J=0$ to $J=2$ followed by radiative decay to $J=1$ with the $J=2 \rightarrow$ 1 lines optically thick. Regions where the anomaly is found probably have $\mathrm{H}_{2}$ densities less than $10^{5} \mathrm{~cm}^{-3}$ and optical depths in the $J=1 \rightarrow 0$ lines greater than 50 .

Subject headings: hyperfine structure - molecules, interstellar

\section{BACKGROUND}

A central question to the interpretation of interstellar molecule observations and the derivation of physical conditions therefrom is whether the transitions are optically thin or whether their excitations are greatly enhanced by trapping of line photons. The two most commonly employed methods of estimating optical depths involve observation of either molecular isotopes of varying abundance or different hyperfine components in a single rotational transition. When hyperfine structure exists, the second method is clearly preferable since a priori the isotopic abundance ratios will be unknown. Unfortunately most hyperfine analyses have proceeded from the trial hypothesis that the components are optically thin (Snyder and Buhl 1973; McGee et al. 1973). The hypothesis is treated in a self-fulfilling way by first assuming equal excitation temperatures in the hyperfine transitions and then calculating apparent optical depths from the relative intensities of the lines. The qualitative behavior anticipated in the second step is that if the lines are thin their intensities will vary as the transition strengths, and if they are thick $(\tau>2)$ their intensities will be equal to within 10 percent. This line of reasoning is valid only if the collisional de-excitation rate $C$ is much greater than the spontaneous emission rate $A$. Then the assumption of equal excitation temperatures in the hyperfine transitions is appropriate. Otherwise if $C<A$, the excitation temperatures in the hyperfine transitions may be increased by photon trapping once they become thick; and since each of them will have a different optical depth, their excitation temperatures will be unequal-the hyperfine component with the greatest transition strength generally having the greatest excitation temperature. Observationally the case $C<A$ and $\tau>1$ will appear qualitatively rather similar to the case $C>A$ and

* This research is sponsored by the National Science Foundation grants GP-40768X (JK) and GP-30400-X5 (NS) and by the Office of Naval Research under contract N0014-67-A-0094-0019 (NS). $\tau<1$ : hyperfine components will be seen with their intensities arranged in order of transition strengths.

In the case of the $J=1 \rightarrow 0$ emission of $\mathrm{HCN}$, an additional clue to the opacities of the hyperfine lines might be provided by the anomalous weakness of the $F=1 \rightarrow 1$ component reported by Wannier et al. (1974). The relative transition strengths of the $F=$ $2 \rightarrow 1,1 \rightarrow 1$, and $0 \rightarrow 1$ hyperfine components are 1 , 0.6 , and 0.2. At two positions where the signal-to-noise ratio is good, the ratios of the hyperfine intensities are $1: 0.4: 0.2$; at the third position, in the center of the Orion nebula, it is $1: 0.6: 0.4$. This anomalous weakness of the $F=1 \rightarrow 1$ component is unlikely to be due to collisions that distinguish between hyperfine levels of the same HCN rotational state. Here we propose a natural explanation by way of photon trapping in the $J=2 \rightarrow 1$ transitions combined with collisional excitation from $J=0 \rightarrow 2$.

\section{THEORY}

A priori, one expects the intensity ratio of the $F=$ $1 \rightarrow 1$ component to the $F=2 \rightarrow 1$ component to lie between 0.6 and 1 , which values represent respectively the ratio when the two components are both thin and the ratio when they are both thermalized. If the three hyperfine levels of $J=1$ were populated only by collisions from $J=0 \rightarrow 1$, the anomalous weakness of the $F=1 \rightarrow 1$ component would be difficult to understand in terms of ordinary collisional excitation and radiative trapping. However, recent calculations by Green and Thaddeus (1974) indicate that $J=0 \rightarrow 2$ collisions are about 6 times more frequent than $J=$ ()$\rightarrow 1$ collisions. Thus the population of $J=1$ can be supplied largely by collisional excitation from $J=0$ to $J=2$ with subsequent radiative decay to $J=1$. Based on this aspect of the collisional rates found by Green and Thaddeus (1974), we wish to demonstrate that the weakness of the $J=1 \rightarrow 0, F=1 \rightarrow 1$ hyperfine component is a result of radiative trapping in the $J=2 \rightarrow 1$ lines. 


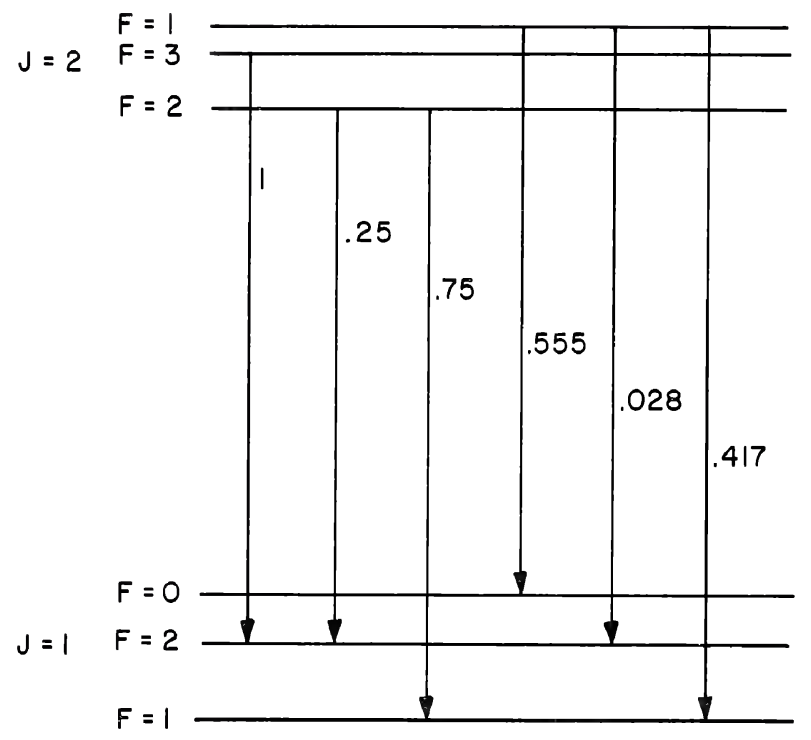

$J=O \quad F=1$

FIG. 1.-Part of the energy level diagram of $\mathrm{HCN}$ (not to scale). The relative spontaneous emission rates for the different hyperfine transitions of $J=2 \rightarrow 1$ are indicated.

Figure 1 illustrates the energy levels (not to scale) of $\mathrm{HCN}$ up to $J=2$. The relative spontaneous decay rates for the different hyperfine transitions of $J=2 \rightarrow$ 1 are also indicated. If radiative de-excitation of $J=2$ is more rapid than collisional de-excitation, most of the population input from $J=0$ to $J=2$ by collisions will be transferred to $J=1$. The collisional input from $J=0$ to the three hyperfine levels of $J=2$ are proportional to the level degeneracies. Then, if the hyperfine transitions of $J=2 \rightarrow 1$ are all thin, the subsequent radiative decay of $J=2$ will distribute population into the three hyperfine levels of $J=1, F=2,1$, and 0 , in the ratio of their degeneracies, or 1:0.6:0.2. When these transitions become thick, however, their line strengths no longer influence the relative rates of population into the $J=1$ hyperfine levels. The net rate of decay, $A / \tau$, where $A$ is the spontaneous emission rate and $\tau$ the opacity, is independent of line strength. Thus, in contrast to the thin case where threequarters of the population input into $J=2, F=2$ decays to $F=1$ and only one-quarter to $F=2$, the thick case can ideally give equal decay rates into $F=2$ and $F=1$. Based on this simple reasoning, we expect trapping in the $J=2 \rightarrow 1$ lines to enhance population into $F=2$ of $J=1$ relative to $F=1$.

An analytical treatment of the excitation processcollisional excitation from $J=0$ to $J=2$ followed by radiative cascade - is straightforward provided one may neglect the population in $J=2$ when calculating the line opacities. Letting $\alpha, \beta$, and $\gamma$ denote the relative population per sublevel in $J=1 ; F=2,1$, and 0 ; we find that the population input into these levels are in the ratios

$$
\begin{aligned}
& 7+\frac{5 \beta}{\alpha+\beta}+\frac{3 \beta \gamma}{\alpha \gamma+\beta \gamma+\alpha \beta}: \frac{5 \alpha}{\alpha+\beta} \\
& +\frac{3 \alpha \gamma}{\alpha \gamma+\beta \gamma+\alpha \beta}: \frac{3 \alpha \beta}{\alpha \gamma+\beta \gamma+\alpha \beta},
\end{aligned}
$$

when the $J=2 \rightarrow 1$ transitions are all optically thick.

We can estimate $\alpha, \beta$, and $\gamma$ from the observed $J=1 \rightarrow 0$ hyperfine intensities. When the $J=1 \rightarrow 0$ lines are thick (which is expected if the $J=2 \rightarrow 1$ lines are thick), the observed intensity of each hyperfine component reflects the excitation temperature of that transition, as

$$
\frac{T_{A}}{T_{0}}+\frac{1}{\exp \left(T_{0} / 2.7\right)-1}=\frac{n_{u} / g_{u}}{n_{l} / g_{l}-n_{u} / g_{u}} .
$$

Here $T_{A}$ is the excess (above the cosmic background) specific intensity in Rayleigh-Jeans temperature units, and $T_{0}=h \nu / k=4.25^{\circ} \mathrm{K}$. The population in the upper level of the transition $(J=1 ; F=2,1$, or 0$)$ is denoted by $n_{u}$, and that in the lower level $(J=0, F=1)$ by $n_{l}$. The corresponding degeneracies are $g_{u}$ and $g_{l}$. From equation (2) and the data of Wannier et al. (1974) at the $2^{\prime} \mathrm{N}$ position, we find $\alpha: \beta: \gamma=1.64: 1.37: 1$. With these relative populations per sublevel in $J=1$, the excitation process discussed above gives relative rates into the three hyperfine levels of $J=1$ of $1: 0.37: 0.13$. Similarly, in the limit $T_{A} \gg T_{0}, \alpha \approx \beta \approx \gamma$ and equation (1) indicates that the decay rates into $J=1$ will be $1: 0.33: 0.1$. In both the observed case and a relevant limiting case the proposed excitation cycle gives an input into $J=1, F=1$ considerably less than 60 percent of that into $J=1, F=2$.

In analogy to the above discussion, one can calculate how collisional excitation from $J=1$ to upper rotational levels $J=2$ and $J=3$ and the subsequent cascade back to $J=1$ would distribute the population of the three $J=1$ hyperfine levels. To include all these processes and the $2.7^{\circ} \mathrm{K}$ background radiation, as well as to obtain the range of molecular density and resulting line intensities over which the $J=1 \rightarrow 0, F=1 \rightarrow 1$ component is weak, we have performed numerical calculations on the excitation of five rotational levels of $\mathrm{HCN}$. We obtain the collisional rates from Green and Thaddeus (1974) and follow the procedure of Scoville and Solomon (1974) and Goldreich and Kwan (1974) in calculating the equilibrium populations. A spherical cloud geometry with a constant radial velocity gradient is adopted. The emitted intensities, in excess of the $2.7^{\circ} \mathrm{K}$ cosmic radiation, are converted to equivalent Rayleigh-Jeans temperatures $\left(T_{A}\right)$ as observed by a perfect antenna. These results are presented in figure 2 in terms of contours of $T_{A}$ of the strongest hyperfine component $(F=2 \rightarrow 1)$ in the $\left(\mathrm{H}_{2}, \mathrm{HCN}\right)$-plane, and of the intensity ratios of the two weaker components to the strongest one.

As expected, the computations show hyperfine in- 


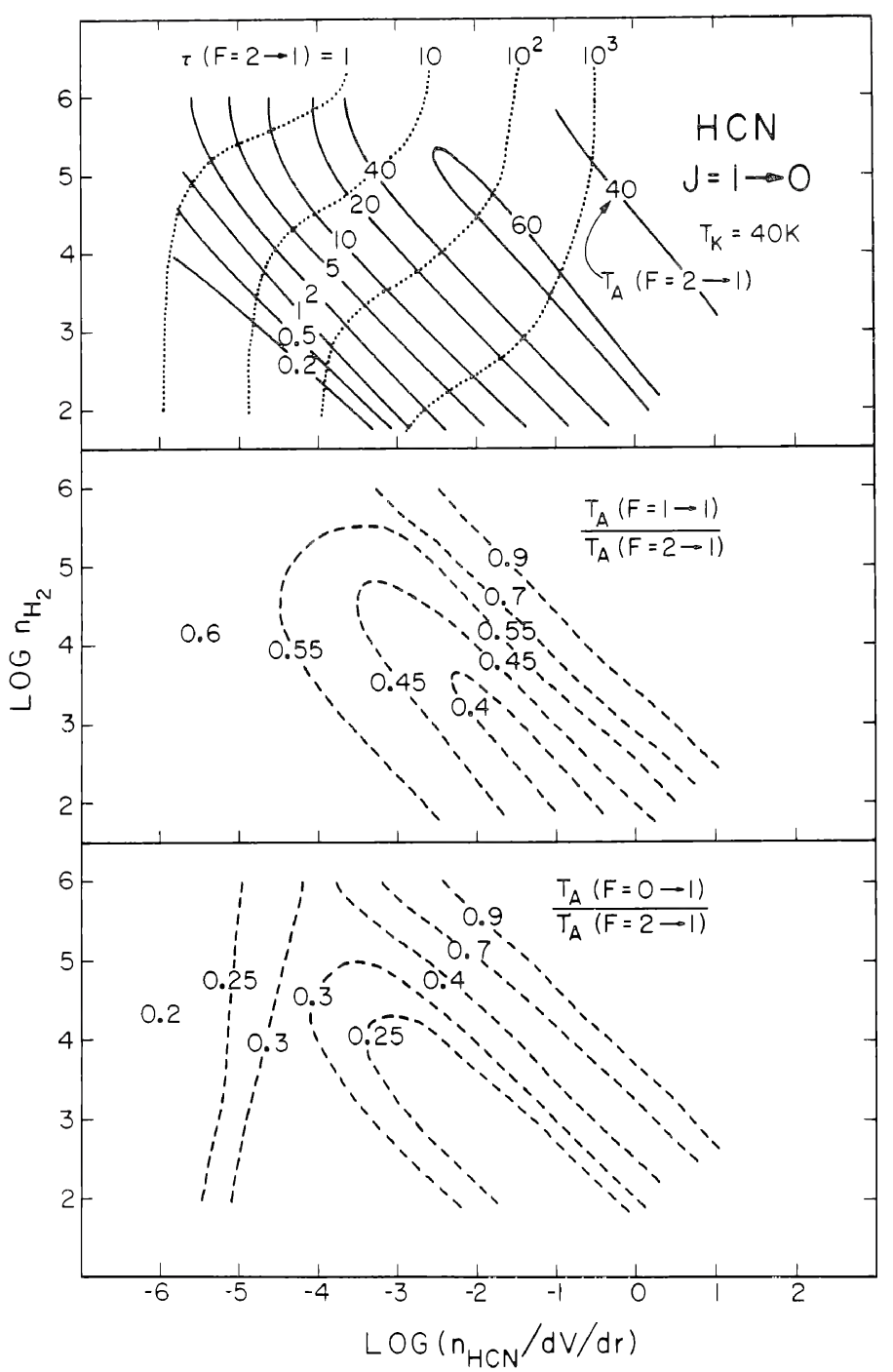

FIG. 2.-Contours of $T_{A}$ (the specific intensity above the cosmic background in Rayleigh-Jeans temperature units) of the hyperfine component $J=1 \rightarrow 0, F=2 \rightarrow 1$, and of the intensity ratios of $F=1 \rightarrow 1$ and $F=0 \rightarrow 1$ to $F=2 \rightarrow 1$. The coordinates $n_{\mathrm{H} 2}$, $n_{\mathrm{HCN}} /$ $(d V / d r)$ have units of $\mathrm{cm}^{-3}$ and $\mathrm{cm}^{-3} /\left(\mathrm{km} \mathrm{s}^{-1} \mathrm{pc}^{-1}\right)$. The collisional rates used correspond to a kinetic temperature of $40^{\circ} \mathrm{K}$.

tensity ratios equal to the line strength ratios $(0.6$ and 0.2 ) when the transitions are optically thin, and equal hyperfine intensities when the transitions are approximately thermalized. Between these two extremes we find quite a complex variation in the ratios. Except for $n_{H_{2}}>10^{6} \mathrm{~cm}^{-3}$, the ratios do not show a monotonic increase from 0.6 and 0.2 to unity as the $\mathrm{HCN}$ density increases. Over a large region of the $\left(\mathrm{H}_{2}, \mathrm{HCN}\right)$-plane, we find a strong decrease from 0.6 in the ratio of the $F=1 \rightarrow 1$ intensity to the $F=2 \rightarrow 1$ intensity. This anomaly occurs in precisely the intensity range where the observed anomaly occurs. Also, over the same region, the intensity ratio of the $F=0 \rightarrow 1$ component to the $F=2 \rightarrow 1$ component remains at 0.2 or is greater, as is observed. The observations of Wannier et al. (1974) at the $2^{\prime} \mathrm{N}$ position are best produced at hydrogen densities less than $\sim 10^{4} \mathrm{~cm}^{-3}$ and greater than $300 \mathrm{~cm}^{-3}$. The lower limit is obtained by restricting the $\mathrm{HCN}$ abundance to be less than $10^{-4}$ of hydrogen and limiting the radial velocity gradient $d V / d r$ to be at least $1 \mathrm{~km} \mathrm{~s} \mathrm{pc}^{-1}$. Bounded by these two limits on the hydrogen density, the locus of a constant intensity ratio of $F=1 \rightarrow 1$ to $F=2 \rightarrow 1$ of 0.4 covers a range of $\mathrm{HCN}$ densities such that the optical depths in $F=$ $2 \rightarrow 1$ is 80 to 1000 . We can conclude from our theory that where the $F=1 \rightarrow 1$ component is weak the hydrogen density is not more than $10^{5} \mathrm{~cm}^{-3}$, the abundance of $\mathrm{HCN}$ between $10^{-5}$ and $3 \times 10^{-8}$ that of $\mathrm{H}_{2}$, and both the $J=1 \rightarrow 0$ and $J=2 \rightarrow 1$ lines are very thick.

That the calculations do not give as low an intensity ratio of $F=0 \rightarrow 1$ to $F=2 \rightarrow 1$ as we obtain in the two illustrative cases is due to the effects of the cosmic background radiation and of collisions from $J=1$ up to $J=3$ and $J=2$. The background radiation tends to equalize the population per sublevel of each hyperfine level. With reference to figure 1 , population will be transferred between $F=0$ and $F=2$ of $J=1$ via 
radiative interactions with the background radiation at the two lines $J=1, F=0 \rightarrow J=2, F=1$, and $J=1, F=2 \rightarrow J=2, F=1$. When the two transitions are thick, the rate of absorption of population from each of the levels $F=0$, and $F=2$ of $J=1$ is the same. Of the amount absorbed from $F=2$, a fraction $\alpha \beta /(\alpha \gamma+\beta \gamma+\alpha \beta)$ is transferred to $F=0$, whereas of the same amount absorbed from $F=0$, a fraction $\beta \gamma /(\alpha \gamma+\beta \gamma+\alpha \beta)$ is transferred to $F=2$. Because $\alpha \geq \gamma$, there will be a net flow of population from $F=2$ to $F=0$. Similarly, because $\beta \geq \gamma$, there will be a net population transfer from $F=1$ to $F=0$. Thus, due to this process, the $F=0$ level always gains population. On the other hand, the $F=1$ level may or may not gain population depending on the relative rate of population input from $F=2$ to the rate of population output to $F=0$. Collisions from $J=1$ up to $J=3$ and $J=2$ and the subsequent cascade would also favor population transfer from $F=2$ and $F=1$ into $F=0$, because the large excess of population per sublevel in the first two levels $(\alpha>\beta>\gamma)$ lead to more collisional excitations from them. A simple demonstration of this process for the more dominant collisions $J=1 \rightarrow 3$ is not possible because the cascade $J=$ $3 \rightarrow 2$ depends on the relative population per sublevel in the hyperfine levels of $J=2$.

The suprathermal intensities which are predicted result from the greater rate of $J=0 \rightarrow 2$ than $J=$ $0 \rightarrow 1$ collisions combined with a more rapid radiative decay rate in $J=2 \rightarrow 1$ than in $J=1 \rightarrow 0$. The suprathermal excitation is quenched when the collisional de-excitation of $J=2$ back to $J=0$ becomes as rapid as the radiative decay to $J=1$.
Our explanation for the weakness of the $F=1 \rightarrow 1$ component relies on the collisional excitations having a large quadruple interaction. Purely dipole collisions will not produce the observed anomaly, because the $J=1$ hyperfine levels will be populated only by direct collisions from $J=0$. Collisions from $J=1$ to $J=2$ followed by radiative decay will redistribute the population unevenly among the three $J=1$ hyperfine levels, when the $J=2 \rightarrow 1$ lines are thick. But by the time the collisional rate from $J=1$ to $J=2$ is high enough that the $J=1$ populations do not immediately decay back to $J=0$, the collisional de-excitation rate of $J=1 \rightarrow 0$ is also high and the $J=1 \rightarrow 0$ transitions begin to be collisionally dominated. Although the anomaly does require $\Delta J=2$ collisions, it does not require the sixfold favoring which Green and Thaddeus (1974) calculate. The effect is still strong if the favoring is only threefold but does almost vanish when $\Delta J \geq 2$ collisional rates are equal to those of $\Delta J=1$. Thus the results here may reaffirm the qualitative behavior of the collisional rates. Since the favoring of $\Delta J=2$ collisions is only mildly dependent on temperature, we feel the adopted kinetic temperature of $40^{\circ} \mathrm{K}$ contributes little uncertainty.

The proposed effect due to trapping in the $J=2 \rightarrow 1$ lines is general and can be readily applied to other molecules with hyperfine structure and large $\Delta J=2$ collisions, possibly DCN (Wilson et al. 1973) and HCCCN (McGee et al. 1973).

We thank Dr. A. Moffet and an unknown referee for helpful comments on the manuscript.

\section{REFERENCES}

Goldreich, P., and Kwan, J. 1974, Ap. J., 189, 441.

Green, S., and Thaddeus, P. 1974, A p. J., 191, 653

McGee, R. X., Newton, L. M., Batchelor, R. A., and Kerr, A. R. 1973, A p. Letters, 13, 25.

Scoville, N. Z., and Solomon, P. M. 1974, Ap.J. (Letters), 187, L67.

John Kwan: Institute for Advanced Study, Princeton, NJ 08540

N. Z. Scoville: Department of Astronomy, California Institute of Technology, Pasadena, CA 91109
Snyder, L. E., and Buhl, D. 1973, A p. J. (Letters), 185, L79.

Wannier, P. G., Encrenaz, P. J., Wilson, R. W., and Penzias, A. A. $1974, A$ p.J. (Letters), 190, L77.

Wilson, R. W., Penzias, A. A., Jefferts, K. B., and Solomon, P. M. 1973, Ap.J. (Letters), 179, L107. 